\title{
UNE ÉTUDE SUR LES FONCTIONS SOUSHARMONIQUES ET SES APPLICATIONS AUX FONCTIONS HOLOMORPHES
}

\author{
Par \\ Masao INOUE
}

(Reçu le 3 juillet, 1942)

\section{Introduction}

La théorie des fonctions analytiques progresse de jour en jour et ses fruits sont déjà abondants. Cependant, malgré son étendue vaste d'une part et sa précision remarquable d'autre part, il y a encore bien des choses à espérer et réfléchir. Par exemple, la manque de la méthode systématique. À cause de cela, on se trouve souvent troublé de la complexité de quelques démonstrations et de la non-solidarité de techniques employées. On demande donc trouver une méthode de l'unification de la théorie des fonctions. À ce point de vue, les occupations de MM. Nevantinna $[11,12]$ et AHLFors $[1,2,3]$ sont très importantes. Et plus tard, on a vu paraître de beaux travaux de MM. RIESZ [17], Privaloff [14, 15, 16] et BeuRling [5] qui partent chacun de l'étude des fonctions harmoniques ou sousharmoniques au de la théorie du potentiel et ont réussi à traiter systématiquement de certains problèmes.

Prenons en considération le travail de ce dernier auteur. Il a présenté dans sa thèse mémoriale une méthode très utile reposée sur le principe de la majoration harmonique pour l'appliquer à l'étude des fonctions holomorphes. Mais il reste encore des choses à améliorer, surtout dans ses techniques. En effet, l'on trouve dans sa thèse des démonstrations trop artificielles et compliquées qui paraissent manquées de vrais outils, et quelques lignes où celleslà causent l'incompréhension du raisonnement. 
En cette circonstance, je souligne une belle idée de M. BRELOT [7], à l'aide de laquelle il ạ démontré, d'une manière très élégante, un théorème de majoration harmonique et l'a appliqué à la limitation du module des fonctions holomorphes. La méthode introduite par lui est basée sur la représentation potentielle des fonctions sousharmoniques d'après $\mathrm{F}$. RIESz et la généralisation maxima du problème de DiRICHLET. On pourra s'attendre à forte raison à ce que son idée va jouer un rôle actif dans les recherches des fonctions sousharmoniques et des fonctions holomorphes. Ainsi j'insiste avec lui sur la nécessité de reprendre non seulement la thèse de BEuRLing mais quelques exposés de la théorie des fonctions holomorphes sur une étude plus systématique en utilisant des résultats plus puissants sur les fonctions sousharmoniques.

Dans le premier Chapitre de ce mémoire $\left({ }^{1}\right)$, je me suis borné à donner une esquisse de l'idée de BRELOT haut dite par présenter son théorème sous un énoncé un peu général avec une application directe, laissant son développement pour un autre travail. Dans le second Chapitre, j'introduis une inégalité de BEuRLING que j'appelle fondamentale. De cette inégalité unique, nous tirons facilement et systématiquement quelques résultats assez intéressants sur les fonctions sousharmoniques, auquèl le troisième Chapitre est consacré. Ces résultáts s'appliquent directement aux fonctions holomorphes. Cela tient, en effet, à ce que, $F(z)$ étant une fonction holomorphe, la fonction $|F(z)|$ est de classe $P L$ [9]; en disant plus précisément, $\log |F(z)|, \log ^{+}|F(z)|$ et $|F(z)|$ sont sousharmoniques. En traduisant des propositions du troisième Chapitre dans la langage de la théorie des fonctions d'une variable complexe, nous énumérons dans le quatrième Chapitre des théorèmes nouveaux, parallèles ou plus ou moins étendus de propositions classiques où intervient le module des fonctions holomorphes $\left({ }^{2}\right)$.

\section{Une esquisse de l'idée de M. BRElot}

1. Théorème de Brelot. Commençons d'abord par expliquer la fonction de WIENER et la notion de résolubilité introduites par M. BRElot [6]: Soit sur la frontière $F$ d'un domaine plan $D$, une fonction $f$ égale en chaque point à un nombre fini, à $+\infty$

(1) Toutes les fonctions considérées dans ce mémoire sont supposées uniformes.

(2) Les chiffres entre crochets renvoient à la liste de bibliographie de la p. 44 . 
(on écrira simplemént $\infty$ ) ou à $-\infty$. On considère la famille (5) des fonctions sousharmoniques $\left({ }^{3}\right)$ dans $D$ dont chacune est bornée supérieurement, et de p. g. 1. (cela signifie la plus grande limite) $\leqq f$ à la frontière. Si (S) est non vide, son enveloppe supérieure $T(x, y)$ vaut dans $D, \infty$ ou une fonction harmonique. On définira alors $\underline{H}_{P}^{P}(x, y)$ en posant:

$$
\begin{array}{ll}
H_{f}^{D}(x, y)=-\infty & \text { si } \text { (S) est vide, } \\
\underline{H}_{f}^{D}(x, y)=T(x, y) & \text { si } \text { (S) est non vide. }
\end{array}
$$

On pourra introduire $\bar{H}_{f}^{D}(x, y)$ par la considération de la même famille relative à $(-f)$. Si $\underline{H}_{f}^{D}$ et $\bar{H}_{f}^{D}$ sơnt partout finies et égales, $f$ sera résolutive et la valeur commune, harmonique, notéé par $H_{f}^{D}$ sera dite fonction de WIENER pour $D, f\left({ }^{4}\right)$. il suit:

Cela dit, le théorème de BrELot [7] peut être énoncé comme

Considérons dans un domaine circulaire plan $Q$ de frontière $\Gamma$, de centre $O$, rayon $R$, une fonction sousharmonique $U \leqq 0$. Soit $\varphi(r)$ sa borne inférieure sur la circonférence de centre $O$ et de rayon $r(0 \leqq r<R)$. Introduisons le domaine $\delta$ déduit de $\Omega$ par. suppression d'un rayon $O A$ et la distribution-frontière $\psi(r)$ égale $\dot{a} 0$ sur $\Gamma^{\prime}$ et $\varphi(O M)$ sur $O A$. Alors elle est résolutive et la fonction de WIENER $H_{\psi}^{\delta}(M)$ majore $U(M)$ sur le rayon $O B$ opposé à $O A$.

Une remarque simple qu'un sous-domaine d'harmonicité d'une fonction sousharmonique n'est pas chargé de masses dans sa représentation potentielle, nous permet de préciser ce théorème comme il suit:

Supposons de plus que $U(z)$ soit en particulier harmonique dans un sous-domaine $D$ de $\Omega . \quad E$, désignant la projection circulaire de $\bar{\Omega}-D$ par rapport à l'origine sur l'axe positif réel $\left({ }^{5}\right)$, introduisons le domaine $\Delta\left({ }^{6}\right)$ déduit de $\Omega$ par suppression de l'ensemble $E$. Alors la fonction $\phi(r)$ considérée comme fonction-distribution sur la frontière de $\Delta$ est résolutive et on a dans $\Omega$

(3) La définition de la fonction sousharmonique est donnée dans mon mémoire [9]

(4) Si $f$ est continue, $f$ est résolutive et $H_{f}^{D}$ fournit la solution du problème généralisé de Dirichlet pour $D, f$.

(5) C'est-à-dire, $E$ est l'ensemble de points de l'axe positif réel que parcourt le module du point $z$ lorsque $z$ parcourt l'ensemble de points du cercle $|z| \leqq R$ n'appartenant pas à $D$.

(6) Nous l'appelons avec Beurling domaine associé à $D$ (par rapport à $\Omega$ ). 


$$
U(z) \leqq H_{\psi}^{\Delta}(-|z|)
$$

Mais on va présenter ce théorème sous l'énoncé suivant qui pourra être considéré plus général $\left({ }^{7}\right)$.

Considérons dans une couronne $Q: 0 \leqq R_{0}<|z|<R_{1}$, une fonction sousharmonique $U(z)$ telle 'que, $z^{\prime}$ designant un point-frontière de $\Omega$,

et

$$
\varlimsup_{z \rightarrow z^{\prime}} U(z) \leqq A \quad \text { sur le contour intérieur } \Gamma_{0} \text { de } \Omega
$$

$$
\varlimsup_{z \rightarrow z^{\prime}} U(z) \leqq B \quad \text { sur le contour extérieur } \Gamma_{1} \text { de } \Omega,
$$

où $A$ et $B$ sont deux constantes finies. Supposons d'ailleurs que $U(z)$ soit harmonique \ans un sous-domaine $D$ de $\Omega$. E désignant la projection circulaire de $\bar{\Omega}-D$ par rapport à l'origine sur l'axe positif réel, introduisons le domaine 4 déduit de $\Omega$ par suppression de l'ensemble $E$ et la distribution-frontière $\psi(r)$ telle que

$$
\begin{array}{rlrl}
\psi(r) & =A & & \text { pour } r=R_{0} \\
& =\text { Bor. inf. } U(z) & \text { pour } R_{0}<r<R \\
& =B & & \text { poù } r=R_{1} .
\end{array}
$$

Alors $\psi(r)$ est résolutive et on a en tout point $z$ de. $\Omega$

$$
U(z) \leqq H_{\psi}^{\Delta}(-|z|) \text {. }
$$

D'abord un lemme.

Soit $G(z, \zeta)$ la fonction de GREEN d'une couronne $\Omega$ : $0<R_{0}<|z|<R_{1}<\infty$, dont le pôle est $\zeta$. Alors, sur la circonférence $|z|=r$, la fonction $G(z, R)$ admet son. maximum en $z=r$ et son minimum en $z=-r$, où $R_{0}<r<R_{1}, R_{0}<R<R_{1}$ et $r \neq R$.

En effet, $G(z, R)$ s'écrit sous la forme

$$
G(z, R)=\log \frac{1}{r_{z}}-H(z), .
$$

$r_{z}$ désignant la distance à $R$ de $z$ et $H(z)$ la solution du problème de Dirichlet pour $Q, \log \frac{1}{r_{z}}$. Prenons deux points distincts $z_{1}$ et $z_{2}$ sur la circonférence $|z|=r$, de sort que $r_{z_{1}}<r_{z_{2}}$, et formons la différence entre $G\left(z_{1}, R\right)$ et $G\left(z_{2}, R\right)$ :

$$
G\left(z_{1}, R\right)-G\left(z_{2}, R\right)=\log \frac{r_{z_{2}}}{r_{z_{1}}}-\left[H\left(z_{1}\right)-H\left(z_{2}\right)\right] \text {. }
$$

(7) Le cas de $R_{0}=0$ se ramène facilement au cas de BRELOT. 
D'après la formule de représentation de la solution du problème de Dirichlét par l'intégral de Stieltjes, on peut écrire

$$
H\left(z_{1}\right)=\int_{C} \log \frac{1}{r_{z}} \omega_{z_{1}}(d z)
$$

et

$$
H\left(z_{2}\right)=\int_{C} \log \frac{1}{r_{z}} \omega_{z_{2}}(d z)
$$

où $C$ désigne les deux contours de $\Omega$ et $\omega_{z_{i}}(d z)$. est la mesure harmonique en $z_{i}$ de $d z$. D'où il vient

$$
H\left(z_{1}\right)-H\left(z_{2}\right)=\int_{C} \log \frac{r_{z^{\prime}}}{r_{z}} \omega_{z_{1}}(d z),
$$

où $z^{\prime}$ désigne le point symétrique à $z$ par rapport à la ligne droite $L$ passant par l'origine et allant perpendiculaire à celle joignant $z_{1}$ et $z_{2}$. Par cette expression, on sait que $H\left(z_{1}\right)-H\left(z_{2}\right)$ vaut la valeur en $z_{1}$ de la solution du problème de DiRICHLET pour $D, \log \frac{r_{z^{\prime}}}{r_{z}}$, soit $U(z)$. Il importe de noter que $U(z)$ s'annule sur $L$.

Or, la fonction de $z, \log \frac{r_{z}}{r_{z^{\prime}}}$, est sousharmonique dans la demicouronne $Q^{\prime}$ limitée par $L$ et $\stackrel{z}{C}^{\prime}$ qui contient les points $z_{1}$ et $R$, de plus harmonique sauf en $R$, et s'annule sur $L$. Par conséquent.

$$
\log \frac{r_{z^{\prime}}}{r_{z}}-U(z)>0
$$

dạns $\Omega^{\prime}$, d'où

$$
G\left(z_{1}, R\right)-G\left(z_{2}, R\right)>0
$$

ce qui met en évidence le lemme.

Ce lemme établi, pour la démonstration du théorème haut énoncé, nous suiverons fidèlement le raisonnement de BRELOT. À cet effet, en cas de $R_{0}>0$, considérons la fonction harmonique dans $\Omega$ :

$$
V(z)=U(z)-W(z)
$$

où

$$
W(z)=\frac{(A-B) \log |z|-A \log R_{0}+B \log R_{1}}{\log \frac{R_{1}}{R_{0}}} .
$$

a) Supposons tout d'abord $V$ bornée, harmonique au voisinage de la frontière et s'annulant sur $\Gamma_{0}$ et $\Gamma_{1}$. Alors à $V(z)$ correspond, 
d'après F. RIEsz, une distribution de masses $\leqq 0$ dans $\Omega$, soit $\nu$, telle que

$$
V(z)=\int_{\Omega} G(\zeta, z) d \nu(\zeta)
$$

Rabattons les masses $\nu$ sur le segment ouvert $\left(R_{0}, R_{1}\right)$ de sorte que chaque segment contenu dans $\left(R_{0}, R_{1}\right)$ porte de la masse comprise entre les circonférences passant par les extrémités, ce qui donne une distribution $\nu^{\prime}$ sur $\left(R_{0}, R_{1}\right)$. Remarquons ici qu'un segment de $\left(R_{0}, R_{1}\right)$ ne portera pas de masses s'il ne contient pas de points de $E$; ce qui est clair d'après la remarque faite au début de ce paragraphe. Or, d'après le lemme, $G(\zeta, z)$, quand $z$ est fixé dans $\Omega$ et $\zeta$ variable sur une circonférence de centre $O$ admet son maximum au point situé sur le rayon liant $O$ et $z$, et son minimum au point symétrique.

.Cela étant, on a pour $R_{0}<r<R_{1}$

$$
\int_{E} G(\zeta,-r) d \nu^{\prime}(\zeta) \geqq \int_{\Omega} G(\zeta,-r) d \nu(\zeta)=V(-r) ;
$$

d'autre part, $z^{\prime}$ étant un point quelconque sur la circonférence $|z|=r$,

$$
\int_{E} G(\zeta, r) d \nu^{\prime}(\zeta) \leqq \int_{\Omega} G\left(\zeta, z^{\prime}\right) d \nu(\zeta)=V\left(z^{\prime}\right)
$$

En prenant $z^{\prime}$ de manière que $V\left(z^{\prime}\right)<\underset{\text { Bor. inf. }}{|z|=r} . \quad V(z)+\varepsilon=\psi(r)-W(r)$ $+\varepsilon$ avec $\varepsilon>0$ arbitraire, on voit que

$$
\text { , } \int_{E}(\zeta, r) d \nu^{\prime}(\zeta) \leqq \psi(r)-W(r)+\varepsilon
$$

Ainsi $\int_{E} G(\zeta, z) d \nu^{\prime}(\zeta)$ est sousharmonique dans $\Omega$, harmonique dans $\Delta, \leqq 0$ et majorée par $\psi(z)-W(z)$ sur $\left(R_{0}, R_{1}\right)$. Par suite, $\psi$ étant bornée par hypothèse, de plus, borélienne par définition, elle est résolutive $\left.{ }^{8}\right)$ et on a dans $\Delta$

$$
\int_{E}^{G}(\zeta, z) d \nu^{\prime}(\zeta) \leqq H_{\psi}^{\Delta}(z)-W(z) .
$$

D'où il résulte que, pour $R_{0}<r<R_{1}$,

$$
U(-r) \leqq \int_{E} G(\zeta,-r) d \nu^{\prime}(\zeta)+W(-r) \leqq H_{\psi}^{\Delta}(-r) .
$$

Or, partant d'une nouvelle fonction $U^{*}(z)=U\left(z e^{i \theta}\right)$, où $\theta$ est une constante entre 0 et $2 \pi$, on arrive de même à l'inégalité:

(8) Voir [6], p. 152. 


$$
U^{*}(-r) \leqq H_{u}^{\Delta}(-r) \quad \text { pour } R_{0}<r<R_{1} .
$$

On en conclut en tout point $z$ de $\Omega$

$$
U(z) \leqq H_{u}^{\Delta}(-|z|) \text {. }
$$

b) Si $V \leqq 0$ est seulement bornée (mais $R_{0}>0$ ), remplaçons dans $\Omega$, d'une part, à l'intérieur de la circonférence $\Gamma_{0}^{(n)}$ : $|z|=R_{0}+\frac{1}{n}, V$ par la fonction de WIENER pour cette couronne, la valeur 0 sur $\Gamma_{0}$ et les valeurs de $V$ sur $\Gamma_{0}^{(n)}$ et, d'autre part, à l'extérieur de la circonférence de $\Gamma_{1}^{(n)}:|z|=R_{1}-\frac{1}{n}, V$ par la fonction de WIENER pour cette couronne, la valeur 0 sur $\Gamma_{1}^{\prime}$ et les valeurs de $V$ sur $\Gamma_{1}^{(n)}$ (pour $n$ suffisamment grand). La nouvelle fonction, soit $V_{n}$, est sousharmonique, car en tout point $z$ de $I_{0}^{(n)}$ (ou $\left.\Gamma_{1}^{(n)}\right), V_{n}(z)$ est inférieure ou égale à la moyenne des valeurs de $V_{n}$ sur toute circonférence $|z|=r$, assez petite, et d'ailleurs p.g.l. de $V_{n}$ est $\leqq V\left({ }^{9}\right)$ sur $\Gamma_{0}^{(n)}$ (ou $\Gamma_{1}^{(n)}$ ). De plus, $V_{n}$ est harmonique au voisinage de la frontière de $\Omega$, s'annule sur la frontière et tend en décroissant vers $V$. Par l'application du raisonnement précédent à cette fonction $V_{n}$, et puis par un passage à la limite, il vient

$$
U(z) \leqq H_{u}^{\Delta}(-|z|) \text {. }
$$

c) On écartera enfin l'hypothèse que $V$ est bornee, en considérant l'enveloppe supérieure $V_{(n)}$ de $V$ et $(-n)$ et passant à la limite. Si sur la frontière de $\Delta, \psi_{(n)}$ correspond à $V_{n}(z)$, on a

$$
-U(z) \leqq V_{(n)}(z)+W(z) \leqq H_{{ }_{(}(n)}^{\Delta}(-|z|) \text {. }
$$

Comme on peut choisir toujours un point $z$ tel que $U(z)$ soit finie, $\psi=\lim _{n \rightarrow \infty} \psi_{(n)}$ est résolutive et on a à la limite

$$
U(z) \leqq H_{u}^{\Delta}(-|z|) .
$$

Le cas de $R_{0}=0$ se traitera comme cas limite de ceux de $R_{0}>0, R_{0} \rightarrow 0$. Ainsi s'achève la démonstration du théorème.

. 2. Démonstration d'un théorème de BEurling. Comme une application du théorème de BRELOT, nous nous proposons de prouver le théorème suivant dû essentiellement à BEURLING [5].

Soit $U(z)$ une fonction sousharmonique dans un domaine $D$ qui est contenu dans un cercle $\Omega:|z|<R$. Supposons que l'on ait en tout point-frontière $z^{\prime}$

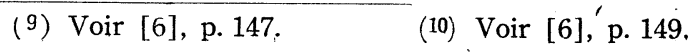




$$
\varlimsup_{z \rightarrow z^{\prime}} U(z) \leqq f^{\prime}\left(\left|z^{\prime}\right|\right),
$$

où $f(r)$ est une fonction convexe de $\log r$ définie dans l'intervalle fermé $[0, R]$, telle que $-\infty<f(0) \leqq f(0+0)<\infty$ et $f(R-0) \leqq f(R)$ $<\infty$. On aura alors

$$
U(z) \leqq H_{f}^{\Delta}(-|z|),
$$

où $\Delta$ est le domaine associé à $D$ par rapport à $\Omega$.

Choisissons une suite de domaines réguliers pour le problème de Dirichlet de manière que

$$
D \supset \cdots \supset D_{n} \supset D_{n-1} \quad \text { et } \quad D_{n} \rightarrow D \text {. }
$$

La fonction $f(z)$ de la variable complexe $z$, de façon que

$$
\begin{array}{ll}
f(z)^{\prime}=f(|z|) & \text { si }|z|>0, \\
f(z)=f(0+0) & \text { si } z=0,
\end{array}
$$

étant sousharmonique bornée supérieurement dans $\Omega$, la fonction $f_{K}(z)$, enveloppe supérieure de $f(z)$ et $f(R)[1+K \log |z| / R]$ l'est aussi, $K$ étant un nombre positif.

Par suite, la fonction $\left(H_{f_{K}}^{D_{n}}, f_{K}\right)$ est encore sousharmonique dans $\Omega$, où

$$
\begin{array}{rlrl}
\left(H_{f_{K}}^{Q_{n}}, f_{K}\right) & =H_{f_{K}}^{D_{n}} \quad \text { dans } & D_{n} \\
= & =f_{K} & \text { dans } & \Omega-D_{n} .
\end{array}
$$

$\varphi_{K}^{(n)}(r)$ étant la borne inférieure de $\left(H_{f_{K}}^{D_{n}}, f_{K}\right)$ sur la circonférence $|z|=r$, elle est égale à $f_{K}(r)$ sur la projection circulaire de $\bar{\Omega}-D_{n}$. D'après le théorème de BRELot, on a l'inégalité

$$
H_{f_{K}}^{D_{n}}(z) \leqq H_{\left.\Phi_{K}^{\prime}\right)}^{\Delta_{n}}(-|z|)=H_{f_{K}}^{\downarrow_{n}}(-|z|) \leqq H_{f_{K}}^{\Delta}(-|z|),
$$

où $\Delta_{n}$ désigne le domaine associé à $D_{n}$.

D'autre part, $\Theta_{K}$ désignant l'enveloppe supérieure de $U$ et $f(R)[1+K \log |z| / R]$,

$$
U_{K}(z) \leqq H_{f_{K}}^{D}(z)
$$

car, en tout point-frontière régulier $z^{\prime}$ de $D$,

$$
\lim _{z \rightarrow z^{\prime}} H_{f_{K}}^{D}(z)=f_{K}(|z|) \geqq \varlimsup_{z \rightarrow z^{\prime}} U_{K}(z) .
$$

En passant à la limite, on obtient finalement

c. q.f. d.

$$
U(z) \leqq \lim _{K \rightarrow \infty} U_{K}(z) \leqq \lim _{K \rightarrow \infty} H_{f_{K}}^{\Delta}(-\mid z !)=H_{f}^{\Delta}(-|z|),
$$


Dans sa thèse, A. BEurLing a demontré un théorème analogue dans quelques conditions moins restrictives. Cependant, son énoncé étant effroyable et la démonstration trop artificielle, il parait que le problème est mal posé. Or, l'on n'hesitera pas à reconnaître que le théorème haut énoncé est déduit, d'une manière très naturelle, du théorème de BRELOT sous une forme moins générale que celle de BEURLIng mais beacoup simple. Ainsi on verra maintenant que le problème est bien posé.

Remarquons que si la projection circulaire de $\bar{\Omega}-D$ contient un segment aboutissant à l'origine, on pourra remplacer la condition $-\infty<f(0) \leqq f(0+0)<\infty$ dans l'énoncé du théorème par $-\infty \leqq f(0)=f(0+0)<\infty$.

Pour le vérifier, dans le raisonnment précédent, posons

$f_{K}=$ enveloppe supérieure de $f,-K$ et $f(R)[1+K \log |z| / R]$ et

$U_{K}=$ enveloppe supérieure de $U,-K$ et $f(R)[1+K \log |z| / R]$.

Alors la démonstration s'achève sans changement.

On pourra présenter le théorème sous l'énoncé suivant considéré plus général.

Soit $U(z)$ une fonction sousharmonique dans un domaine $D$ qui est contenu dans une couronne $\Omega: 0 \leqq R_{0}<|z|<R_{1}<\infty$. Supposons que l'on ait en tout point-frontière $z^{\prime}$

$$
\varlimsup_{z \rightarrow z^{\prime}} U(z) \leqq f\left(\left|z^{\prime}\right|\right),
$$

où $f(r)$ est une fonction convexe de log $\dot{r}$ définie dans l'intervalle férmé $\left[R_{0}, R_{1}\right]$, telle que $-\infty<f\left(R_{0}\right) \leqq f\left(R_{0}+0\right)<\infty$ et $f\left(R_{1}-0\right) \leqq$ $f\left(R_{1}\right)<\infty$. On aura alors

$$
U(z) \leqq H_{f}^{\Delta}(-|z|),
$$

où $\Delta$ est le domaine associé à $D$ par rapport à $Q\left({ }^{11}\right)$.

La même remarque s'applique aussi à ce théorème. En effet, si la projection circulaire de $\bar{\Omega}-D$ contient un segment aboutissant à $R_{0}$, on peut remplacer la condition $-\infty<f\left(R_{0}\right) \leqq f\left(R_{0}+0\right)<\infty$ dans l'énoncé du théorème par $-\infty \leqq f\left(R_{0}\right)=f\left(R_{0}+0\right)<\infty\left({ }^{12}\right)$.

(11) Dan's le cas de $R_{0}=0$, posons $f(0)=f(0+0)$ en considérant $f(z)$ dans $|z|<R$, lorsque on prend le cercle $|z|<R$ au lieu de $\Omega$. ।

(12) $\mathrm{Si}-\infty<f\left(R_{0}\right)$, on peut admettre que $f\left(R_{0}\right) \leqq f\left(R_{0}+0\right)<\infty$. 


\section{Une inégalité fondamentale}

1. Notion de la densité logarithmique. Considérons un ensemble linéaire $E$ supposé mesurable et situé sur l'axe positif réel que nous désignerons par $I$. La mesure logarithmique de $E$ sera définie par

$$
m E=\int_{\mathrm{I}} \frac{h(t)}{t} d t
$$

où $h(t)$ est la fonction caractéristique de $E$.

En désignant par $E(r, R)$ la portion de $E$ contenue dans l'intervalle $[r, R]$, posons

$$
\begin{aligned}
& \bar{\mu} E=\varlimsup_{R \rightarrow \infty} \frac{m E(r, R)}{\log R} ; \\
& \mu E=\varlimsup_{R \rightarrow \infty} \frac{m E(r, R)}{\log R},
\end{aligned}
$$

$r$ étant $>0$. Ces deux quantités $\bar{\mu} E$ et $\mu E$ ainsi définies indépendamment de $r>0$ s'appellent respectivement densité logarithmique supérieure et densité logarithmique inférieure de $E$ et satisfont aux relations suivantes:

$$
0 \leqq \mu E \leqq \bar{\mu} E \leqq 1
$$

et

$$
\bar{\mu} E+\mu(I-E)=1 \text {. }
$$

Il est bon de rappeler ici des relations entre cette notion et celle de la densité ordinaire de $E$. La mesure ordinaire de $E$ étant donnée par

$$
m^{*} E=\int_{\mathrm{I}} h(t) d t
$$

les densités supérieure et inférieure de $E$ seront par définition

$$
\begin{aligned}
& \bar{\mu}^{*} E=\varlimsup_{R \rightarrow \infty} \frac{m^{*} E(r, R)}{R} ; \\
& \mu^{*} E=\varlimsup_{R \rightarrow \infty} \frac{m^{*} E(r, R)}{R} .
\end{aligned}
$$

Comme tout à l'heure,

$$
0 \leqq \mu^{*} E \leqq \bar{\mu}^{*} E \leqq 1
$$

et

$$
\bar{\mu}^{*} E+\mu^{*}(I-E)=1
$$


Nous nous contentons de signaler les inégalités importantes $\left({ }^{13}\right)$

$$
\mu^{*} E \leqq \mu E \leqq \bar{\mu} E^{*} \leqq \bar{\mu}^{*} E .
$$

2. Inégalité fondamentale. Nous indiquons maintenant une inégalité très importante qui joue un rôle fondamental dans ce qui va suivre. Expliquons auparavant des notations qui seront employées fréquemment dans la suite. Nous désignons par $D_{R}$ la portion ouverte d'un domaine $D$ contenue dans le 'cercle $\Omega_{R}$ : $|z|<R$, par $E(R)$ la projection circulaire de $\overline{\Omega_{R}}-D_{R}$ par rapport à l'origine sur l'axe positif réel, par $E(r, R)$ la portion de $E(R)$ contenue dans l'intervalle fermé $[r, R]$, par $\Delta_{R}$ le domaine associé à $D_{R}$ par rapport à $\Omega_{R}$, par $\psi_{R}(t)$ la fonction égale à 0 pour $0 \leqq t<R$ et 1 pour $t=R$, et adoptons la brève notation $E=E(D)$ $=E(\infty)$. Nous employons dorénavant ces notations sans explication.

Ceci posé, on aura la limitation suivante :

$$
H_{\psi_{R}}^{\Delta_{R}}(-r) \leqq \frac{4}{\pi} \operatorname{arctg} \sqrt{\frac{2 R^{2}}{R^{2}+r^{2}}} e^{-\frac{1}{2} m E(r, R)}<2 e^{-\frac{1}{2} m E(r, R)},
$$

où $0 \leqq r<R$.

En effet, si $E(R)$ est formé par un nombre fini de segments, la formule est bien connue ([5], p. 64). Donc il s'agit à présent de la vérifier dans le cas général. À cet effet, formons une suite de $E_{n}(R)$ dont. chacun est formé par un nombre fini de segments fermés de sorte que $E_{n}(R) \supseteqq E(R)$ et $E_{n}(R) \rightarrow E(R)$. On a alors

$$
\begin{aligned}
H_{\psi_{R}}^{\Delta_{R}^{(n)}}(-r) & \leqq \frac{4}{\pi} \operatorname{arctg} \sqrt{\frac{2 R^{2}}{R^{2}+r^{2}}} e^{-\frac{1}{2} m E_{n}(r, R)} \\
& \leqq \frac{4}{\pi} \operatorname{arctg} \sqrt{\frac{2 R^{2}}{R^{2}+r^{2}}} e^{-\frac{1}{2} m E(r, R)}<2 e^{-\frac{1}{2} m E(r, R)},
\end{aligned}
$$

où $\Delta_{R}^{(n)}$ désigne le domaine déduit de $\Omega_{R}$ par suppression de $E_{n}(R)$. En égard à la relation aisée à vérifier: $\lim _{n \rightarrow \infty} H_{\psi_{R}}^{\Delta V_{R}^{(n)}}(z)=H_{\psi_{R}}^{\Delta R}(z)\left({ }^{14}\right)$,

(13) La démonstration s'achève sans peine, par exemple, en calquant sur celle de Dinghas [8].

(14) $z^{\prime}$ étant un point-frontièrè régulier de $D_{R}$, il l'est aussi pour $\Delta_{R}^{(n)}$. On a donc $\lim _{z \rightarrow z^{\prime}} H_{\underset{R}{R}}^{\Delta(n:}=0$ pour tout point-frontière régulier $z^{\prime}$ de $\Delta_{R}$. D'autre part, $H_{\psi R}^{\Delta(n)}$ étant non décroissante et majorée toujours par $H_{\psi_{R}}^{\Delta_{R}}$, la fonction limite de lá suite $H_{\psi}^{\Delta(n)}$ est harmonique dans $\Delta_{R}$, et prend 1 sur $|z| \stackrel{\psi_{R}}{=} R$, sauf en $z=R$, et 0 en d'autres points-frontière réguliers de $\Delta_{R}$, ce qui montre que cette fonction est identique à $H_{\bigcup_{R}}^{\Delta_{R}}$ 
on aura à la limite l'inégalité proposée.

Il convient d'ailleurs de donner pour les applications suivantes une autre formule qui sera déduite immédiatement du théorème de BRELOT et de l'inégalité précédente.

Soit $U(z)$ une fonction sousharmonique dans $D_{R}$ qui satisfait aux conditions suivantes: $z^{\lambda}$ étant un point-frontière de $D_{R}\left({ }^{15}\right)$,

$$
\begin{array}{ll}
\varlimsup_{z \rightarrow z^{\prime}} U(z) \leqq A & \text { pour }\left|z^{\prime}\right|=R, \\
\varlimsup_{z \rightarrow z^{\prime}} U(z) \leqq B<A & \text { pour }\left|z^{\prime}\right|<R,
\end{array}
$$

où $A$ et $B$ sont deux constantes finies.

Cela étant, l'inégalité que j'appelle fondamentale est la suivante:

$$
U(z)<B+2(A-B) e^{-\frac{1}{2} m E(|z|, R)} .
$$

En effet, d'après le théorème de BrELOT et l'inégalité précédente,

$$
\frac{U(z)-B}{A-B} \leqq H_{\psi_{R}}^{\Delta_{R}}(-|z|)<2 e^{-\frac{1}{2} m E(|z|, R)} .
$$

de là, on tire l'inégalité mentionnée.

\section{Résultats sur les fonctions sousharmoniques}

1. Théorèmes du type de PHRAgMÉN-LINDEl̨ÖF. Considérons un domaine infini $D$, ayant $z=\infty$ comme point-frontière, et une fonction sousharmonique $U(z)$ dans $D$ telle que l'on ait

$$
\varlimsup_{z \rightarrow z^{\prime}} U(z) \leqq M^{\prime}
$$

en tout point-frontière $z^{\prime} \grave{a}$ distance finie, $M$ étant une constante finie.

Désignons par $M(r)$ la borne supérieure de $U(z)$ sur la portion de la circonférence $|z|=r$ qui est contenue dans $D$. Il s'agit alors de chercher quelques restrictions sur la croissance de $M(r)$ qui entraînent dans $D$ l'inégalité

$$
U(z) \leqq M \text {. }
$$

Commençons tout d'abord par démontrer le théorème suivant.

(15) Dès lors, nous désignerons toujours par $z^{\prime}$ un point-frontière quelconque d'un domaine considéré. 
Théorème 1. S'il existe un nombre non négatif $\rho(\leqq 1 / 2)$ tel que l'on ait

on a dans $D$

$$
\varlimsup_{r \rightarrow \infty} \frac{M(r)}{r^{p}} \leqq 0 \text { et } \lim _{r \rightarrow \infty}[2 \rho \log r-m E(1, r)]<\infty,
$$

$$
U(z) \leqq M
$$

Démonstration. D’après la première condition, $\bar{\varepsilon}>0$ étant donné arbitrairement petit, on a pour tout $R$ assez grand

$$
M(R)<\varepsilon R^{\dot{\rho}} \text {. }
$$

On n'a qu'à considérer le cas où $\rho>0$ et $M<\varepsilon R^{\circ}$ pour tout $R$ suffisamment grand, car le théorème est évident dans le cas contraire.

En appliquant l'inégalité fondamentale établie tout à l'heure aux $A=\varepsilon R^{\rho}$ et $B=M$, on obtient dans $D_{R}$

$$
\begin{aligned}
U(z) & <M+2\left(\varepsilon R^{\rho}-M\right) e^{-\frac{1}{2} m E(|z|, R)} \\
& =M+2 \varepsilon e^{\rho \log R-\frac{1}{2} m E(|z|, R)}-2 M e^{-\frac{1}{2} m E(|z|, R)} .
\end{aligned}
$$

En y tendant $R$ vers $\infty$ de manière que la seconde condition soit réalisée, ،

$$
U(z)<M+\varepsilon K
$$

où $K$ est une constante finie. D'où finalement

$$
U(z) \leqq M
$$

D'une manière analogue, il est aisé de prouver le

'Théorème 2." S'il existe un nombre non négatif $\rho(\leqq 1 / 2)$ tel l'on ait

on a dans $D$

$$
\lim _{r \rightarrow \infty} \frac{M(r)}{r^{p}} \leqq 0 \text { et } \varlimsup_{r \rightarrow \infty}[2 \rho \log r-m E(1, r)]<\infty,
$$

$$
U(z) \leqq M .
$$

Citons encore deux théorèmes analogues aux précédents.

Théorème 3. S'il existe un nombre non négatif $\rho(<1 / 2)$ tel l'on ait

$$
\varlimsup_{r \rightarrow \infty} \frac{M(r)}{r^{p}}<\infty \text { et } 2 \rho<\bar{\mu} E
$$

on a dans $D$.

$$
U(z) \leqq M
$$


Théorème 4. S'il existe un nombre non négatif $\rho(<1 / 2)$ tel l'on ait

$$
\lim _{r \rightarrow \infty} \frac{M(r)}{r^{p}}<\infty \text { et } 2 \rho<\mu E
$$

on a dans $D$

$$
U(z) \leqq M \text {. }
$$

Nous nous bornons à prouver le derriier théorème, car il n'y a rien de différence essentielle.

D'après la première condition, on peut trouver une constante positive $K$ et une suite de valeurs positives de $\boldsymbol{r}_{\boldsymbol{i}}$ tendant vers $\infty$ pour avoir

$$
M\left(r_{i}\right)<K r_{i}^{p} .
$$

Comme précédemment, il suffira de considérer seulement le cas où $\rho>0$ et $M<K r_{i}^{p}$ pour tout $i$ suffisamment grand. En vertu de l'inégalité fondamentale,

$$
U(z)<M+2 e^{\frac{1}{2}\left[2 \rho-\frac{m E\left(|z|, r_{i}\right)}{\log r_{i}}\right] \log r_{i}}-2 M e^{-\frac{1}{2} m E\left(|z|, r_{i}\right)} .
$$

Si l'on pose $\eta=\underline{\mu} E-2 \rho>0$, on trouve d'après la seconde condition, pour tout $i$ assez grand et pour $z$ fixé arbitrairement dans $D$, que

$$
\mu E-\frac{\eta}{2}<\frac{m E\left(|z|, r_{i}\right)}{\log r_{i}}
$$

Par suite,

$$
U(z)<M+2 K e^{-\frac{\eta}{4} \log r_{i}}-2 M e^{-\frac{1}{2} m E\left(|z|, r_{i}\right)} .
$$

$\mu E$ étant positive, le deriner membre de cette inégalité tend vers 0 quand $r_{i}$ tend vers $\infty$ d'une manière quelconque. Par conséquent,

$$
U(z) \leqq M
$$

Il y a lieu de signaler ici que si l'on transforme le domaine $D$ par l'inversion pour obtenir un domaine $D^{\prime}$ dont l'origine est un point-frontière. Les résultats haut acquis réponderont au problème suivant: Soit $U^{\prime}(z)$ une fonction sousharmonique dans $D^{\prime}$. Supposons que $\varlimsup_{z \rightarrow z^{\prime}} U^{\prime}(z) \leqq M$ en tout point-frontière à l'exception de l'origine. Quelle condition est-elle suffisante pour affirmer que $U^{\prime}(z) \leqq M$ dans $D^{\prime}$ ? Cela revient au même: Que peut-on dire de 
l'ordre de croissance de $M(r)$ au voisinage de l'origine dans le cas où $U^{\prime}(z)$ n'est pas bornée dans ce voisinage? La transposition sera immédiate.

2. Cas de domaines particuliers. Notons que les propositions haut obtenues peuvent évidemment être précisées dans chaque cas de domaines particuliers.

Nous nous plaçons dans les hypothèses du $n^{\circ}$ précédent. Soit de plus le domaine $D$ contenu dans un angle (pour fixer les idées; $0<\theta<\pi / \alpha$ où $1 / 2<\alpha)$. Dans ce cas, la seule condition

$$
\lim _{r \rightarrow \infty} \frac{M(r)}{r^{\alpha}} \leqq 0
$$

est suffisante pour qu'on ait dans $D$

$$
U(z) \leqq M .
$$

En effet, si l'on considère $U^{*}(z)=U\left(z^{\frac{1}{2^{\alpha}}}\right)^{(16)}$ au lieu de $U(z)$ dans le raisonnement précédent, la transcription de la condition impusée nous conduit à la suivante :

$$
\lim _{r \rightarrow \infty} \frac{M^{*}(r)}{r^{\frac{1}{2}}} \leqq 0
$$

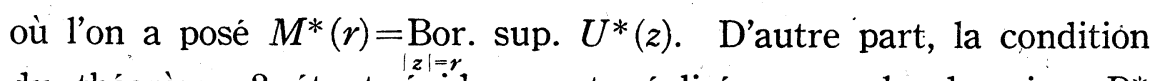
$\mathrm{du}$ théorème 2 étant évidemment réalisée pour le domaine $D^{*}$ transformé de $D$ par la représentation $z^{2 \alpha}$; on obtient d'après le théorème 2

$$
U^{*}\left(2^{2 \alpha}\right)=U(z) \leqq M
$$

Nous allons plus avant en étudiant de plus près un tel cas particulier. À cet effet, d'une manière générale, considérons le cas oú un domaine $D$ est limité (ou limité partiellement) par au moins une courbe continue partant de l'origine et s'étendant à l'infini, et supposons la frontière de $D$ régulière pour le problème de DIRICHLET.

Désignons par $\bar{\omega}$ la densité logarithmique supérieure de l'ensemble fermé des valeurs de $r$ telles qu'on ait $\theta(r) \leqq \pi / \alpha, \theta(r)$ étant la mesure angulaire des parties de la circonférence $|z|=r$ qui sont contenues dans $D$ et $\alpha$ un nombre positif fini $\geqq 1 / 2$. Admettons que $\bar{\omega}>0$ et choisissons un nombre $\omega$ de manière que $\bar{\omega}>\omega>0$.

Ceci fait, sous l'hypothèse

(16) On précisera cette transformation tout à l'heure. 


$$
\varlimsup_{r \rightarrow \infty} \frac{M(r)}{r^{\alpha \omega}}<\infty,
$$

nous allons montrer que $U(z)$ reste inférieure ou égale à̀ $M$ dans $D$.

En effet, d'après l'hypothèse, il existe deux nombres positifs finis $K$ et $R_{0}$ tels que

$$
\dot{M}(\boldsymbol{r})<K \boldsymbol{r}^{\alpha \omega}
$$

pour tout $r>R_{0}$. On n'a qu'à considérer comme précédemment le cas où $M<K r^{\alpha \omega}$.

Désignons par $U_{R}(z)$ la fonction harmonique dans $D_{R}$ qui prend $K R^{\alpha \omega}$ sur la partie-frontière de $D_{R}$ où $|z|=R$ et $M$ sur le reste de la frontière. Évidemment $U(z) \leqq U_{R}(z)$ dans $D_{R}$. Et, considérons la fonction $U_{R}^{*}(z)$ définie dans $D_{R}^{*}$ par la manière suivante: $D_{R}^{*}$ désignant le domaine formé par les $z^{2 \alpha}$ lorsque les $z$ parcourent $D_{R}$ (il va sans dire qu'on prend une branche convenanble de la fonction $z^{2 \alpha}$, soit $T$ cette transformation correspondante), la valeur de $U_{R}^{*}(z)$ en $z$ de $D_{R}^{*}$ vaut le maximum de celles de $U_{R}$ en $z^{1 / 2 \alpha}$ de $D_{R}$ qui correspondent au $z$ par $T\left({ }^{17}\right)$.

On vérifie alors sans difficulté que:

1) $U_{R}^{*}(z)$ est sousharmonique uniforme et continue dans $D_{R}^{*}$;

2) $U(z) \leqq U_{R}(z) \leqq U_{R}^{*}\left(z^{2 \alpha}\right)$ dans $D_{R}$;

3) $\varlimsup_{z \rightarrow z^{\prime}} U_{R}^{*}(z)=M$ en tout point-frontière $z^{\prime}$ de $D_{R}^{*}$, tel que $\left|z^{\prime}\right|<R^{2 \alpha}$, et $\varlimsup_{z \rightarrow z^{\prime}} U_{R}^{*}(z) \leqq K R^{\alpha \omega}$ en tout point-frontière $z^{\prime}$ de $D_{R}^{*}$, tel que $\left|z^{\prime}\right|=R^{2 x^{2}}$.

En observant d'ailleurs que la densité logarithmique supérieure (ou inférieure) est invariante par la transformation $z^{2 \alpha}$, on voit que

$$
\bar{\mu} E^{*}=\bar{\omega},
$$

où $E^{*}$ désigne $E\left(D^{*}\right), D^{*}$ étant le domaine limite de $D_{R}^{*}$ pour $R \rightarrow \infty$. Alors, d'après l'inégalité fondamentale,

$$
\begin{aligned}
U(z) & \leqq U_{R}^{*}\left(z^{2 \alpha}\right)<M+2\left(K R^{\alpha \omega}-M\right) e^{-\frac{1}{2} m E^{*}\left(|z|^{2 \alpha}, R^{2 \alpha}\right)} \\
& =M+2 K e^{\left[\omega-\frac{m E^{*}\left(|z|^{2 \alpha}, R^{2 \alpha}\right)}{\log R^{2 \alpha}}\right] \log R^{\alpha}}-2 M e^{-\frac{1}{2} m E^{*}\left(|z|^{2 \alpha}, R^{2 \alpha}\right)} .
\end{aligned}
$$

À cause de $\bar{\omega}>0$,

$$
\lim _{R \rightarrow \infty} e^{-\frac{1}{2} m E^{*}\left(\left.|z|\right|^{2 \alpha}, R^{2 \alpha}\right)}=0 .
$$

(17) Le domaine $D_{R}^{*}$ s'obtient en projetant sur un seul feuillet les points du domaine transformé de $D_{R}$ par $T$, qui peut bien se recouvrir partiellement. 
Par conséquent, en faisant tendre $R$ vers $\infty$ de sorte que

$$
\omega-\frac{m E^{*}\left(|z|^{2 \alpha}, R^{2 \alpha}\right)}{\log R^{2 \alpha}} \rightarrow \omega-\bar{\omega}<0,
$$

par suite

$$
\lim _{R \rightarrow \infty} e^{\left[\omega-\frac{m E^{*}\left(\left.|z|\right|^{2 \alpha}, R^{2 \alpha}\right)}{\log R^{2 \alpha}}\right] \log R^{\alpha}}=0
$$

on a dans $D$

$$
U(z) \leqq M
$$

Nous obtenons ainsi le

Théorème 5. 'Si l'ón a

$$
\varlimsup_{r \rightarrow \infty} \frac{M(r)}{r^{\alpha \omega}}<\infty
$$

pour un nombre $\omega$ tel que $\bar{\omega}>\omega>0$, on a dans $D$

$$
U(z) \leqq M .
$$

Désignant de même par $\underline{\omega}$ la densité logarithmique inférieure de l'ensemble des valeurs de $r$ telles que $\theta(r) \leqq \pi / \alpha, 1 / 2 \leqq \alpha$, il est aisé de démontrer par un raisonnement analogue le

Théorème 6. Si l'on a

$$
\lim _{r \rightarrow \infty} \frac{M(r)}{r^{\alpha \omega}}<\infty
$$

pour un nombre $\omega$ tel que $\underline{\omega}>\omega>0$, on a dans $D$

$$
U(z) \leqq M .
$$

Dans le théorème 5 (ou 6 ), la condition pourra être remplacée par

$$
\begin{aligned}
& , \varlimsup_{r \rightarrow \infty} \frac{M(r)}{r^{\alpha \omega}} \leqq 0 \text { et } \lim _{r \rightarrow \infty}\left[2 \alpha \log r-m E\left(1, r^{2 \alpha}\right)\right]<\infty \\
& \text { (ou } \lim _{r \rightarrow \infty} \frac{M(r)}{r^{\alpha \omega}} \leqq 0 \text { et } \varlimsup_{r \rightarrow \infty}\left[2 \alpha \log r-m E\left(1, r^{2 \alpha}\right)\right]<\infty \text { ). }
\end{aligned}
$$

3. Extensions. Considérons maintenant dans un domaine infini $D$ diu type considéré jusqu'ici une fonction sousharmonique $U(z)$ telle que l'on ait en tout point-frontière $z^{\prime}$ à distance finie,

$$
\varlimsup_{z \rightarrow z^{\prime}} U(z) \leqq f\left(\left|z^{\prime}\right|\right),
$$


où $f(r)$ est une fonction non décroissante de $r$ qui prend des valeurs finies pour $0 \leqq r<\infty$. Nous nous occupons de chercher une formule générale de limitation de $U(z)$.

Supposons d'abord qu'il existe un nombre non négatif $\rho$ tel que

$$
\varlimsup_{r \rightarrow \infty} \frac{M(r)}{r^{p}}<\infty .
$$

En vertu de cette hypothèse, on peut choisir une constante positive finie $K$ de manière que, pour tout $R$ assez grand,

$$
M(R)<K R^{\circ} \text {. }
$$

Il y a deux cas à distinguer :

1) S'il existe une suite de $R_{i}$ tendant vers $\infty$ de sorte que

$$
K R_{i}^{p} \leqq f\left(R_{i}\right)
$$

on a $M\left(R_{i}\right)<f\left(R_{i}\right)$.

2) Sinon, autrement dit, si pour tout $R$ assez grand

$$
f(R)<K R^{p},
$$

on arrive à une inégalité forte intéressante.

En remarquant que $f(r)$ est hon décroissante et appliquant l'inégalité fondamentale aux $D_{R}, A=K R^{\rho}$ et $B=f(R)$, on aura dans $D_{R}$

$$
U(z)<f(R)+2\left[K R^{\circ}-f(R)\right] e^{-\frac{1}{2} m E(|z|, R)} .
$$

Si $f(R) \geqq 0$ pour tout $R$ assez grand,

$$
U(z)<f(R)+2 K e^{\frac{1}{2}\left[2 p-\frac{m E(|z|, R)}{\log R}\right] \log R} .
$$

En posant $R=r^{\lambda}(\lambda>1)$, il vient pour $|z|=r>1$.

$$
\begin{aligned}
U(z) & <f\left(r^{\lambda}\right)+2 K e^{\frac{1}{2}\left[2 \rho-\frac{m E\left(r, r^{\lambda}\right)}{\log r^{\lambda}}\right] \log r^{\lambda}} \\
& =f\left(r^{\lambda}\right)+2 K e^{\frac{1}{2}\left[2 \rho-\frac{m E\left(1, r^{\lambda}\right)}{\log r^{\lambda}}+\frac{m E(1, r)}{\log r^{\lambda}}\right] \log r^{\lambda}} .
\end{aligned}
$$

$\eta>0$ étant donné arbitrairement, il existe une suite de $r_{i}$ tendant vers $\infty$, telle que

$$
\bar{\mu}-\eta<\frac{m E\left(1, r_{i}^{\lambda}\right)}{\log r_{i}^{\lambda}} \text { et } \frac{m E\left(1, r_{i}\right)}{\log r_{i}}<\bar{\mu}+\eta,
$$

où l'on a écrit pour abréger $\bar{\mu}=\bar{\mu} E$ et écrira de même $\mu=\mu E$ dans la suite. Ainsi 


$$
U(z)<f\left(r_{i}^{\lambda}\right)+2 K e^{\frac{1}{2}\left[2 p-\bar{\mu}+\frac{\bar{\mu}}{\lambda}+\eta\left(1+\frac{1}{\lambda}\right)\right] \log r_{i}^{\lambda}} .
$$

Si $2 \rho<\bar{\mu}$, choisissons $\lambda$ de façon que

$$
2 \rho-\bar{\mu}\left(1-\frac{1}{\lambda}\right)<0 \text {, }
$$

c'est-à-dire que

$$
\lambda>\frac{\bar{\mu}}{\bar{\mu}-2 \rho} \geqq 1 \text {. }
$$

Puis, prenons comme $\eta$ une valeur positive assez petite satisfaisant à l’inégalité

$$
2 \rho-\bar{\mu}+\frac{\bar{\mu}}{\lambda}+\eta\left(1+\frac{1}{\lambda}\right)<0 .
$$

Pour ces deux nombres $\lambda$ et $\eta$ ainsi choisis,

$$
\lim _{r_{i} \rightarrow \infty} 2 K e^{\frac{1}{2}\left[2 p-\bar{\mu}+\frac{\bar{\mu}}{i}+\eta\left(1+\frac{1}{\lambda}\right)\right] \log r_{i}^{\lambda}}=0 .
$$

On a donc pour tout $z$ de $D$ dont le module est égal à $r_{i}$,

$$
U(z)<f\left(|z|^{\lambda}\right)+\varepsilon(|z|),
$$

où $\varepsilon(|z|)$ désigne une quantité tendant vers 0 pour $|z| \rightarrow \infty$.

D'autre part, $\eta>0$ étant donné arbitrairement, on a pour tout $r$ assez grand

$$
\mu-\eta<\frac{m E\left(1, r^{\lambda}\right)}{\log r^{\lambda}} \text { et } \frac{m E(1, r)}{\log r}<\bar{\mu}+\eta
$$

Par suite,

$$
U(z)<f\left(r^{\lambda}\right)+2 K e^{\frac{1}{2}\left[2 \rho-\underline{\mu}+\frac{\bar{\mu}}{\lambda}+n\left(1+\frac{1}{\lambda}\right)\right] \log r^{\lambda}}
$$

Si $2 \rho<\mu$, choisissons $\lambda$ de façon que

$$
2 \rho-\mu+\frac{\bar{\mu}}{\lambda}<0 \text {. }
$$

En effet, il suffit pour cela de prendre $\lambda \cdot$ de manière que

$$
\lambda>\frac{\bar{\mu}}{\mu-2 \rho} \geqq 1 \text {. }
$$

Après cela, attribuons à $\eta$ une valeur positive satisfaisant à l'inégalité

$$
2 \rho-\mu+\frac{\bar{\mu}}{\lambda}+\eta\left(1+\frac{1}{\lambda}\right)<.0
$$


Pour ces deux nombres $\lambda$ et $\eta$ ainsi choisis,

$$
\lim _{r \rightarrow \infty} 2 K e^{\frac{1}{2}\left[2 \rho-\underline{\mu}+\frac{\bar{\mu}}{\lambda}+\eta\left(1+\frac{1}{\lambda}\right)\right] \log r^{\lambda}}=0 .
$$

On a donc pour tout $z$ de $D$

$$
U(z)<f\left(|z|^{\lambda}\right)+\varepsilon(|z|) .
$$

D'après le théorème 3 , les résultats subsistent encore pour le cas où $\lim _{R \rightarrow \infty} f(R)<0$. Mais, en réalité, le cas où $\lim _{R \rightarrow \infty} f(R)<\infty$ n'a aucun intérêt.

Nous résumons les résultats obtenus dans l'énoncé suivant.

Théorème 7. Supposons qué l'on ait $f(r) \leqq M(r)$ pour tout $r$ suffisamment grànd et qu'il existe un nombre non négatif $\rho$ tel que l.on ait

$$
\varlimsup_{r \rightarrow \infty} \frac{M(r)}{r^{p}}<\infty
$$

Si $2 \rho<\bar{\mu}$, on peut trouver, une suite infinie de circonférences $|z|=r_{n}, r_{n} \rightarrow \infty$, sur lesquelles on a pour $\lambda>\frac{\vec{\mu}}{\bar{\mu}-2 \rho}$

$$
U(z)<f\left(|z|^{\lambda}\right)+\varepsilon(|z|) .
$$

D'ailleurs, si $2 \rho<\mu$, on a dans $D$ pour $\lambda>\frac{\bar{\mu}}{\mu-2 \rho}$

$$
U(z)<f\left(|z|^{\lambda}\right)+\varepsilon(|z|),
$$

où $\varepsilon(|z|)$ désigne une quantité tendant vers 0 pour $|z| \rightarrow \infty$.

D'une manière analogue, il est facile d'établir le

Théorème 8. Supposons que l'on ait $f(r) \leqq M(r)$ pour tout $r$ suffisamment grand et qu'il existe un nombre non négatif $\rho$ tel que l'on ait

$$
\lim _{r \rightarrow \infty} \frac{M(r)}{r^{p}}<\infty
$$

Si $20<\mu$, on peut trouver une suite infinie de circonférences $|z|=r_{n}, r_{n} \rightarrow \infty$, sur lesquelles on a pour $\lambda>\frac{\bar{\mu}}{\mu-2 \rho}$

$$
U(z)<f\left(|z|^{\lambda}\right)+\varepsilon(|z|) .
$$

Il convient de présenter ces deux théorèmes sous les énoncés suivants moins générals mais plus commodes pour les applications.

Corollaire 1. Dans les mêmes conditions que celles du théorème 7, on a si $2 \rho<\bar{\mu}$, 


$$
\left.\lim _{r \rightarrow \infty} \frac{\log ^{+} M(r)}{\log r} \leqq \frac{\bar{\mu}}{\bar{\mu}-2 \rho} \varlimsup_{r \rightarrow \infty} \frac{\log ^{+} f(r)}{\log r}{ }^{18}\right)
$$

et d'ailleurs si $2 \rho<\mu$,

$$
\varlimsup_{r \rightarrow \infty} \frac{\log ^{+} M(r)}{\log r} \leqq \frac{\bar{\mu}}{\underline{\mu}-2 \rho} \varlimsup_{r \rightarrow \infty} \frac{\log ^{+} f(r)}{\log r} .
$$

Corollaire 2. Dans les mêmes conditions que celles du théorème 8 , on a si $2 \rho<\psi$,

$$
\varliminf_{r \rightarrow \infty} \frac{\log ^{+} M(r)}{\log r} \leqq \frac{\bar{\mu}}{\mu-2 \rho} \varlimsup_{r \rightarrow \infty} \frac{\log ^{+} f(r)}{\log r} .
$$

4. Applications à l'étude du module minimum des fonctions sousharmoniques.

Il est bon en premier lieu d'introduire la notion des ordres supérieur et inférieur d'une fonction sousharmonique $U(z)$ définie dans un domaine infini $D$. Deux quantités

$$
\bar{\rho}=\varlimsup_{r \rightarrow \infty} \frac{\log ^{+} M(r)}{\log r} \text { et } \varrho=\lim _{r \rightarrow \infty} \frac{\log ^{+} M(r)}{\log r}
$$

s'appellent respectivement ordre supérieur et ordre inférieur de $U(z)$ (dans $D$ ). Il importe de remarquer que les inégalités $\lim M(r) / r^{\circ} \leqq 0$ et $\lim _{r \rightarrow \infty} M(r) / r^{p} \leqq 0$ entrainent respectivement $\bar{\rho} \leqq \rho$ et $\rho \leqq \rho$, et que réciproquement, les inégalités $\bar{\rho}<\rho$ et $\rho<\rho$ entraînent respectivement $\varlimsup_{r \rightarrow \infty} M(r) / r^{p} \leqq 0$ et $\lim _{r \rightarrow \infty} M(r) / r^{p} \leqq 0$.

Considérons dans tout le plan une fonction continue et sousharmonique $U(z) \geqq 0$ d'ordres supérieur $\bar{\rho}$ et inférieur $\varrho$. Soit $U(z)$ non bornée supérieurement. Nous allons démontrer d'abord les théorèmes suivants, où l'on posera

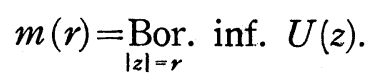

Théorème 9. Si ̄o est inférieur à $1 / 2$, la densité logarithmique inférieure de l'ensemble des valeurs de $r$ telles qu'on ait $m(r)>a$, $a$ étant un nombre fini quelconque, est supérieure ou égale à $1-2 \bar{\rho}$.

Théorème 10. Si $\rho$ est inférieur à $1 / 2$, la densité logarithmique supérieure de l'ensemble des valeurs de $r$ telles qu'on ait $m(r)>a$, a étant iın nombre fini quelconque, est supérieure ou égale à $1-2 \varrho$.

La démonstration du premier étant analogue à celle du second, nous nous bornons à prouver seulement le théorème 10 .

(18) $\log +f$ vaut $\log f$ si $f \geqq 1$ et 0 si $f \leqq 1$. 
À cet effet, choisissons des chemins $\Gamma$-qui peuvent s'être séparés -s'étendant à l'infini et rencontrant toute circonférence $|z|=r$, sur lesquels $U(z)=m(|z|)$. Soit $\Gamma^{\prime}$ la partie fermée de $\Gamma^{\prime}$ où $U(z)=m(|z|) \leqq a$. Considérons $U(z)$ dans le domaine $D$ déduit du plan entier par suppression de $\Gamma^{\prime}$. Il est évident que $U(z) \leqq a$ à la frontière.

Supposons que la densité supérieure de l'ensemble des valeurs de $r$ appartenant à $I-E$, pour lesquelles $m(r)>a$, soit inférieure à $1-2 \varrho$, c'est-à-dire que $\bar{\mu}(I-E)<1-2 \rho$. Alors, en vertu de l'égalité notée p. 24 ,

Or

$$
\mu E>2 \varrho .
$$

$$
\lim _{r \rightarrow \infty} \frac{M(r)}{r^{p \prime}}=0,
$$

où $\rho^{\prime}$ est un nombre positif fini tel que $\mu E>2 \rho^{\prime}>2 \rho$. On obtìent donc d'après le théorème 4

$$
U(z) \leqq a ;
$$

ce qui est contradictoire à l'hypothèse initiale que $U(z)$ n'est pas bornée supérieurement. Le théorème se trouve ainsi démontré.

Ayant égard aux résultats du $n^{\circ}$ précédent, on verra que les théorèmes 9 et 10 sont susceptibles d'une extension évidente. En effet, nous pouvons démontrer le

Théorème 11 $\left({ }^{19}\right)$. Soit $U(z)$ d'ordre supérieur $\bar{\rho}$ moindre que $1 / 2$. $E_{a}$ désignant l'ensemble des valeurs de $r$ telles que $m(r)>M(r)^{a}$, on $a$

et

$$
\bar{\mu} E_{(h-2) \bar{\rho}} \geqq 1-h \bar{\rho}
$$

$$
\mu E_{(h-2) \underline{p}} \geqq 1-h \bar{\rho},
$$

où $h$ est un nombre fini quelconque, supérieur à 2 et inférieur $\grave{a}$ $1 / \bar{\rho}$.

La démonstration est facile. Comme précédemment, choisissons des chemins $\Gamma$ s'étendant à l'infini, sur lesquels $U(z)=m(|z|)$. Soit $\Gamma_{a}$ la partie fẹrmée de $\Gamma$ où $U(z) \leqq M(r)^{a}$. Considérons $U(z)$ dans le domaine $D_{a}$ déduit du plan entier par suppression de $\Gamma_{a}$. Évidemment, $U(z) \leqq M(r)^{a}$ à la frontière.

Supposons d'abord que

(19) Ce théorème est comparable avec celui de Dinghas [8]. 


$$
\bar{\mu} E_{(h-2) \bar{\rho}}<1-h \bar{\rho},
$$

c'est-à-dire que

$$
\mu=\mu\left(I-E_{(h-2) \overline{\mathrm{p}}}\right)>h \bar{\rho} .
$$

$\rho^{\prime}$ étant un nombre positif tel que $\mu>h \rho^{\prime}>h \bar{\rho}$,

$$
\varlimsup_{r \rightarrow \infty} \frac{M(r)}{r^{p \prime}}=0 .
$$

Cela observé, le corollaire 1 s'applique aux $U(z), D_{(h-2) \bar{p}}$ et $f(r)=M(r)^{(h-2) \bar{p}}$, et il donne

$$
\bar{\rho} \leqq \frac{(h-2) \bar{\rho}^{2}}{\mu-2 \rho^{\prime}} .
$$

$\grave{A}$ supposer $\bar{\rho}>0$, on en tire contradictoirement en tenant compte de ce que $\mu-2 \rho^{\prime}>(h-2) \rho^{\prime}$,

$$
1<\frac{\bar{\rho}}{\rho^{\prime}} .
$$

Donc, il faut que

dans le cas où $\bar{\rho}>0$.

$$
\bar{\mu} E_{(h-2) \bar{\rho}} \geqq 1-h \bar{\rho}
$$

Si $\bar{\rho}=0$, d'après le théorème 10 ,

$$
\bar{\mu} E_{(h-2) \overline{\mathrm{p}}}=\bar{\mu} E_{0}=1=1-h \bar{\rho} ;
$$

l'inégalité proposée subsiste donc pour $\bar{\rho}=0$. La première inégalité est ainsi verifiée.

Passons à la seconde inégalité. Si $\varrho=0$, d'aprés le théorème 9 ,

$$
\mu E_{(h-2) \rho}=\mu E_{0} \geqq 1-2 \bar{\rho} \geqq 1-h \bar{\rho} ;
$$

l'inégalité est bien vérifiée.

Dans le cas où $\varrho>0$, supposons que

d'où

$$
\mu E_{(h-2) \varrho}<1-h \bar{\rho},
$$

$$
\bar{\mu}=\bar{\mu}\left(I-E_{(h-2) \underline{\rho}}\right)>h \bar{\rho} .
$$

En choisissant un nombre $\rho^{\prime}$ tel que $\bar{\mu}>h \rho^{\prime}>h \bar{\rho}$, on obtient d'après le corollaire 1 appliqué aux $U(z), D_{(h-2) \rho}$ et $f(r)=M(r)^{(h-2) \rho}$,

$$
\varrho \leqq \frac{(h-2) \rho \bar{\rho}}{\bar{\mu}-2 \rho^{\prime}},
$$

d'où l'inégalité absurde 


$$
1<\frac{\bar{\rho}}{\rho^{\prime}} ;
$$

ce qui achève la démonstration du théorème.

Notons encore le théorème suivant aisé à établir.

Théorème 12. $S_{a}$ désignant l'ensemble des valeurs de $r$ telles que $m(r)>a \log r$, ò̀ a est un nombre positif fini quelconque, on a

et

$$
\bar{\mu} S_{a} \geqq 1-2 \rho
$$

$$
\mu S_{a} \geqq 1-2 \bar{\rho} \text {. }
$$

Pour la démonstration, $a$ étant donné, on considère la fonction

$$
U(z)-a \log |z|
$$

qui est sousharmonique sauf en l'origine. D'après les théorèmes 9 et 10 ,

et

$$
\bar{\mu} S_{a} \geqq 1-2 \varrho^{\prime}
$$

$$
\mu S_{a} \geqq 1-2 \bar{\rho}^{\prime},
$$

où $\bar{\rho}^{\prime}$ et $\varrho^{\prime}$ sont rèspectivement les ordres supérieur et inférieur de $U(z)-a \log |z|$.

Alors, les inégalités évidentes $\bar{\rho}^{\prime} \leqq \bar{\rho}$ et $\varrho^{\prime} \leqq \rho$ entraînent immédiatement

et

$$
\bar{\mu} S_{a} \geqq 1-2 \varrho
$$

\section{c. q. f. d.}

$$
\bar{\mu} S_{a} \geqq 1-2 \bar{\rho},
$$

Signalons finalement une application de ce dernier théorème. Soit $\left\{D_{n}\right\}$ une suite infinie de domaines finis à l'intérieur desquels $U(z)$ reste inférieure à $a \log |z|, a$ étant un nombre fini; $R_{n}$ et $r_{n}$ les bornes supérieure et inferrieure des distances à l'origine des points de $D_{n}$. On supposera que $D_{n}$ soit assez éloigné de l'origine pour $n$ suffisamment grand. D'après le théorème 12 , on sait que

et

$$
\bar{\mu} E\left(\sum^{\infty} E_{n}\right) \leqq 2 \bar{\rho}
$$

$$
\mu E\left(\sum^{\infty} E_{n}\right) \leqq 2 \varrho,
$$

d'où

$$
\grave{\lim }_{n \rightarrow \infty} \frac{\log \frac{R_{n}}{r_{n}}}{\log R_{n}} \leqq 2 \bar{\rho}
$$


et

$$
\lim _{n \rightarrow \infty} \frac{\log \frac{R_{n}}{r_{n}}}{\log R_{n}} \leqq 2 \rho .
$$

Enfin,

$$
1 \leqq \varlimsup_{n \rightarrow \infty} \frac{\log R_{n}}{\log r_{n}} \leqq \frac{1}{1-2 \bar{\rho}} \text { si } \bar{\rho}<\frac{1}{2}
$$

et

$$
1 \leqq \lim _{n \rightarrow \infty} \frac{\log R_{n}}{\log r_{n}} \leqq \frac{1}{1-2 \varrho} \quad \text { si } \varrho<\frac{1}{2}
$$

Dans le cas particulier où $R_{n-1} \leqq r_{n}, n=1,2, \ldots$, on pourra obtenir les inégalités plus précises:

et

$$
1 \leqq \varlimsup_{n \rightarrow \infty} \frac{\log R_{n}}{\log r_{n} \prod_{i=1}^{n-1} \frac{r_{i}}{R_{i}}} \leqq \frac{1}{1-2 \bar{\rho}} \text { si } \bar{\rho}<\frac{1}{2}
$$

$$
1 \leqq \lim _{n \rightarrow \infty} \frac{\log R_{n}}{\log r_{n} \prod_{i=1}^{n-1} \frac{r_{i}}{R_{i}}} \leqq \frac{1}{1-2 \varrho} \text { si } \varrho<\frac{1}{2} .
$$

Les mêmes résultats s'obtiennent pour une suite infinie de domaines finis $\left\{D_{n}\right\}$ à l'intérieur desquels $U(z)$ reste bornée supérieurement $<M$.

En nous plaçant dans cette dernière hypothèse, précisons des résultats dans un cas particulier. Si $M\left(R_{n}\right)<\left(\log R_{n}\right)^{K}, R_{n} \rightarrow \infty$, $n=1,2, \ldots$, on a d'après l'inégalité fondamentale

$$
U(z)<M+2\left[\left(\log R_{n}\right)^{K}-M\right] e^{-\frac{1}{2} \log \frac{R_{n}}{r_{n}}} \text {. }
$$

Pour que $U(z)$ ne soit pas bornée supérieurement dans tout le plan, il faut que

$$
\lim _{n \rightarrow \infty} \frac{r_{n}}{R_{n}}\left(\log R_{n}\right)^{2 K}=\infty .
$$

Si l'on suppose de plus que $R_{n-1} \leqq r_{n}, n=1,2, \ldots$, on aura

$$
\lim _{n \rightarrow \infty}\left(\log R_{n}\right)^{2 K} \prod_{i=1}^{n} \frac{r_{i}}{R_{i}}=\infty .
$$




\section{Applications immédiates aux fonctions holomorphes}

1. Généralisations d'un théorème de PHRAgMén-LindelöF. Considérons une fonction holomorphe $F(z)$ dans un domaine infini $D$ et supposons que l'on ait

$$
\varlimsup_{z \rightarrow z^{\prime}}|F(z)| \leqq M
$$

en tout point-frontière $z^{\prime} \grave{a}$ distance finie, $M$ étant une constante finie.

Comme on l'a déjà indiqué, log $|F(z)|$ est sousharmonique. Les conséquences immédiates des résultats acquis dans II. 1, sont alors les suivantes, où l'on posera

$$
M(r)=\max _{|z|=r} \mid F(z)
$$

Théorème $1^{\prime}$. S'il existe un nombre non négatif $\rho(\leqq 1 / 2)$ tel que l'on ait

$$
\varlimsup_{r \rightarrow \infty} \frac{\log M(r)}{r^{p}} \leqq 0 \text { et } \lim _{r \rightarrow \infty}[2 \rho \log r-m E(1, r)]<\infty,
$$

on a dans $D$

$$
|F(z)| \leqq M
$$

Théorème 2 '. 'S'il existe un nombre non négatif $\rho(\leqq 1 / 2)$.tel que l'on ait

$$
\lim _{r \rightarrow \infty} \frac{\log M(r)}{r^{\rho}} \leqq 0 \text { et } \varlimsup_{r \rightarrow \infty}[2 \rho \log r-m E(1, r)]<\infty
$$

on a dans $D$.

$$
|F(z)| \leqq M
$$

Théorème $3^{\prime}$. S'il existe un nombre non négatif $\rho(<1 / 2)$ tel que l'on ait

$$
\varlimsup_{r \rightarrow \infty} \frac{\log M(r)}{r^{p}}<\infty \text { et } 2 \rho<\bar{\mu} E
$$

on a dans $D$

$$
|F(z)| \leqq M
$$

Théorème 4'. S'il existe un nombre non négatif $\rho(<1 / 2)$ tel que l'on ait

$$
\lim _{r \rightarrow \infty} \frac{\log M(r)}{r^{p}}<\infty \text { et } 2 \rho<\mu E,
$$

on a dans $D$

$$
|F(z)| \leqq M
$$


2. Conséquences de forme des domaines de détermination infinie des fonctions entières. Soit $F(z)$ une-fonction entière non constante. Un domaine infini limité (ou limité partiellement) par au moins une courbe continue s'étendant à l'infini sera dit domaine de détermination infinie de $F(z)$, lorsque cette fonction n'est pas bornée à l'intérieur de ce domaine tandis que le module de $F(z)$ est borné sur la frontière de ce domaine. On défininira les ordres supérieur $\bar{\rho}$ et inférieur $\rho$ de $F(z)$ par

$$
\bar{\rho}=\varlimsup_{r \rightarrow \infty} \frac{\log ^{+} \log ^{+} M(r)}{\log r} \text { et } \rho=\lim _{r \rightarrow \infty} \frac{\log ^{+} \log ^{+} M(r)}{\log r} \text {. }
$$

Ceci rappelé, les théorèmes 5 et 6 donnent lieu aux propositions suivantes.

Théorème 5'. Soit $\Delta$ un domaine de détermination infinie d'une fonction entièré d'ordre supérieur $\bar{\rho}$ moindre que $\rho$. Aloys la densité logarithmique inférieure de l'ensemble des valeurs de $r$ telles que la mesure angulaire des parties de la circonférence $|z|=r$ qui sont contenues dans $\Delta$ soit supérieure $\grave{a} \pi / \rho$ est positive.

Théorème 6'. Soit $\Delta$ un domaine de détermination infinie d'une fonction entière d'ordre inférieur $\rho$ moindre que $\rho$. Alors la densité logarithmique supérieure de l'ensemble des valeurs de $r$ telles que la mesure angulaire des parties de la circonférence $|z|=r$ qui sont contenues dans $\Delta$ soit supérieure $\grave{a} \pi / \rho$ est positive.

Ces deux théorèmes étendant des propositions de Milloux [10] reviennent aux même:

Soit $\Delta$ un domaine de détermination infinie d'une fonction entière. Si l'ensemble des valeurs de $r$ telles que la mesure angulaire des parties de la circonférence $|z|=r$ qui sont contenues dans $\Delta$ soit supérieure à $\pi / \rho$, est de densité logarithmique inférieure (ou supérieure) nulle, l'ordre supérieur (ou inférieur) de cette fonction entière est alors supérieur ou égal à $\rho$.

3. Limitations généraux. Les théorèmes suivants découlent comme conséquences immédiates des théorèmes 7 et 8 .

Soit $F(z)$ une fonction holomorphe dans un domaine infini dont la frontière passe par l'infini, telle que l'on ait

$$
\varlimsup_{z \rightarrow z^{\prime}}|F(z)| \leqq f(|z|),
$$

où $f(r)$ est une fonction non décroissante de $r$ qui prend des valeurs finies. Supposons que 


$$
f(r) \leqq \log M(r)
$$

pour tout $r$ assez grand.

Théorème $7^{\prime}$. Supposons qu'il existe un nombre non négatif $\rho$ tel que l'on ait

$$
\varlimsup_{r \rightarrow \infty} \frac{\log M(r)}{r^{p}}<\infty
$$

Si $2 \rho<\bar{\mu} E$, il existe une suite infinie de circonférences $|z|=r_{n}$, $r_{n} \rightarrow \infty$, sur lesquelles on a

$$
|F(z)|<f\left(|z|^{\lambda}\right)+\varepsilon(|z|) \text { pour } \lambda>\frac{\bar{\mu} E}{\bar{\mu} E-2 \rho} .
$$

D'ailleurs si $2 \rho<\mu E$, on a alors dans $D$

$$
\text { . }|F(z)|<f\left(|z|^{\lambda}\right)+\varepsilon(|z|) \text { pour } \lambda>\frac{\bar{\mu} E}{\mu E-2 \rho},
$$

où $\varepsilon(|z|)$ désigne une quantité tendant vers 0 pour $|z| \rightarrow \infty$ :

Théorème $8^{\prime}$. 'Supposons qu'il existe un nombre non négatif $\rho$ tel que l'on ait

$$
\lim _{r \rightarrow \infty} \frac{\log M(r)}{r^{p}}<\infty
$$

Si $2 \rho<u E$, il existe une suite infinie de circonférences $|z|=r_{n}$, $r_{n} \rightarrow \infty$, sur lesquelles on $a$

$$
|F(z)|<f\left(|z|^{\lambda}\right)+\varepsilon(|z|) \quad \text { pour } \lambda>\frac{\bar{\mu} E}{\mu E-2 \rho} .
$$

4. Extensions d'un théorème de WiMAN. Des théorèmes 5 et 6 , on déduit un théorème de WIMAN aujourd'hui classique, d'après lequel une fonction entière non constante d'ordre supérieur (ou inférieur) moindre que $1 / 2$ n'a pas de chemin asymptotique fini. D'autre part, les théorèmes 9 et 10 en donnent deux autres extensions dues à Dinghas [8].

Théorème 9'. Si l'ord̈re supérieur $\bar{\rho}$ d'une fonction entière non constante est inférieur à $1 / 2$, la densité logarithmique inférieure de l'ensemble des valeurs de $r$ telles que $m(r)=\underset{|z|=r}{\min }|F(z)|>a$, a étant un nombre fini quelconque, est supérieure ou ê égale à $1-2 \bar{\rho}$.

Théorème-10'. Si l'ordre inférieur $\varrho$ d'une fonction entière non constante est inférieur à $1 / 2$, la densité logarithmique supérieuré de 
l'ensemble des valeurs de $r$ telles que $m(r)=\min _{|z|=r}|F(z)|>a$, a étant un nombre fini quelconque, est supérieure ou égale à $1-2 \varrho$.

Mais le théorème 11 nous conduit à une autre bonne extension que voici :

Théorème 11'. Soit $F(z)$ une fonction entière d'ordres supérieur $\bar{\rho}$ et inférieur $\varrho$ moindres que $1 / 2$. $k$ étant un nombre positif fini quelconque, la densité logarithmique supérieure de l'ensemble des valeurs de $r$ telles que

$$
\frac{\log \log m(r)}{\log \log M(r)}>k \dot{\bar{\rho}},
$$

et la densité logarithmique inférieure de l'ensemble des valeurs de $r$ telles que

$$
\frac{\log \log m(r)}{\log \log M(r)}>k \varrho
$$

sont chacune supérieures ou égales à $1-(k+2) \bar{\rho}$.

On pourra comparer ce résultat avec ceux de MM. BEsICoviTCH [4], Pennycuick [13] et Dinghas [8].

Le dernier théorème 12 sera traduit comme il suit.

Théorème 12'. $F(z)$ une fonction entière non constante d'ordres supérieur $\bar{\rho}$ et inférieur $\varrho$. Alors la densité logarithmique supérieure (ou inférieure) de l'ensemble des valeurs de $r$ telles que

$$
\frac{\log \log m(\boldsymbol{x})}{\log r}>a
$$

a étant un nombre fini quelconque, est supérieure ou égale à $1-2 \varrho$ (ou $1-2 \bar{\rho})$.

Terminons ce mémoire en signalant que la dernière considération du Chapitre qui précède nous permettra de donner une démonstration très simple ainsi qu'une extension d'un théorème de VALIRON [18], qui sera obtenue, $F(z)$ étant une fonction entière, en posant $U(z)=\log ^{+}|F(z)|$ dans le raisonnement de la: p. $39\left({ }^{20}\right)$.

(20) Voir aussi Dinghas [8] et Milloux [10]. 


\section{Bibliographie}

[1] L. AHLFORS, 'Über eine Methode in der, Theorie der meromorphen Funktionen, Soc. Sc. Fennicae Comment. Phys.-math., 8 (1932).

[2] - Zur Theorie der Überlagerungsfächen, Acta Math., 65 (1935).

[3] - Über die Anwendung differentialgeometrischer Methoden zur Untersuchung von Überlagerungsflächen, Acta Soc. Sc. Fennicae, 2 (1937).

[4] A. S. Besicovitch, On integral functions of order $<1$, Math. Ann., 97 (1927).

[5] A. BeuRling, Études sur un problème de majoration harmonique, Thèse, Upsal (1933).

[6] M. Brelot, Familles de Perron et problème de Dirichlet, Acta Szeged, 9 (1939).

[7] - Quelques applications aux fonctions holomorphes de la théorie moderne du potentiel et du problème de DiRICHLET, Bull. Soc. Sc. Liége, 6-7 (1936).

[8] A. Dinghas, Über das Minimum einer Klasse von subharmonischen Funktionen, Sitzungsber. Preuss. Akad., Phys.-math., (1937).

[9] M. InOuE, Sur les fonctions dont le logarithme est sousharmonique, Journ. Fac. Sc. Kyūsyū Imp. Univ., 3 (1942).

[10] H. Milloux, Sur les domaines de détermination infinie des fonctions entières, Acta Math., 61 (1933).

[1.1] R. Nevanlinna, Zur Theorie der meromorphen Funktionen, Acta Math., 46 (1925).

[12] --, Eindeutige analytische Funktionen, Berlin, Springer (1937).

[13] K. Pennycuick, On a theorem of Besicovitch, Journ. London Math. Soc., 10 (1935).

[14] I. PRIVAlofF, Sur certaines questions des fonctions harmoniques et subharmoniques et des fonctions analytiques, Rec. math. Moscou, 41 (1935).

[15] - Sur quelques applications de la mesure harmonique des ensembles de points à certains problèmes de la théorie des fonctions, Rec. math. Moscou, N.s. 3 (1938).

[16] - - Sur le principe de maximum généralisé pour les fonctions subharmoniques, C. R. Acad. Sc. URSS., 19 (1931).

[17] F. RIESz, Sur les fonctions subharmoniques et leur rappart à la théorie du potentiel, Part I, Acta Math., 48 (1926)-Part II, Acta Math., 54 (1930).

[18] G. Valiron, Remarque sur un théorème de M. Carleman, C. R., 176 (1923). 